\title{
Manejo da síndrome serotoninérgica no contexto da urgência: uma revisão narrativa
}

\author{
Management of serotonergic syndrome in the context of urgency: a narrative review
}

Manejo del síndrome serotonérgico en el contexto de urgencia: una revisión narrativa

Laryssa de Lacerda Rodrigues ${ }^{4 *}$, Isadora Thamires Pacheco Celestino ${ }^{4}$, André Rodrigues de Senna Batista Filho ${ }^{1}$, Fernanda Bianco Duarte ${ }^{2}$, Flávia Cristina Avelar ${ }^{3}$, Isabella La-Côrte Morais Matos², Lucas Pasqual Boaventura de Santana ${ }^{5}$, Mariana Mesquita Rodarte ${ }^{2}$, Rebeca Mousinho Pestana de Oliveira ${ }^{6}$.

\section{RESUMO}

Objetivo: Esclarecer a clínica da síndrome serotoninérgica e assim possibilitar seu correto diagnóstico, de forma que seu manejo seja feito de maneira precoce, resultando na prevenção significativa da morbimortalidade dessa síndrome. Revisão bibliográfica: A Síndrome Serotoninérgica é caracterizada por uma manifestação medicamentosa adversa gerada por aumento dos níveis de serotonina no Sistema nervoso central e periférico. Por ter manifestações pouco específicas, piora clínica rápida e aumento da prescrição dos medicamentos serotonergicos nas últimas décadas, tornou-se necessário a formulação de critérios diagnósticos para uma identificação e reversão de forma rápida. Contudo, ocorre um desconhecimento médico sobre tal síndrome, corroborando mais para sua subnotificação, o que torna relevante este estudo. Considerações finais: Esta pesquisa revelou que um dos desafios quanto à síndrome supracitada é evitar o seu diagnóstico incorreto, por isso, conhecer os pilares da sua clínica, por meio da educação médica continuada, bem como as principais interações farmacológicas que culminam na doença, é fundamental para obter o diagnóstico correto e, assim, iniciar precocemente as medidas terapêuticas preconizadas, diminuindo a morbimortalidade.

Palavras-chave: Serotonina, Toxicidade, Antagonistas da serotonina.

\section{ABSTRACT}

Objective: To clarify the clinic of the serotoninergic syndrome and to allow its correct diagnosis, in such a way that its management becomes effective in a timely manner, resulting in a significant prevention of the morbidity and mortality of this syndrome. Literature review: Serotoninergic syndrome is characterized as an adverse drug manifestation caused by increased levels of serotonin in the central and peripheral nervous system. Formulation of diagnosis criteria became more necessary because of the nonspecific manifestations of it, rapid clinical worsening and increased prescription of serotoninergic drugs in recent decades. However medical doctors don't have enough knowledge about that syndrome, corroborating with the sub notification of it and making this study more relevant. Final considerations: This investigation revealed that one of the challenges with respect to the aforementioned syndrome is to avoid its incorrect diagnosis, therefore, to know the pillars of its clinic, through continuing medical education, as well as the main pharmacological interactions that culminate. in sickness, it is essential to obtain the correct diagnosis and, therefore, to take the recommended therapeutic measures in time, reducing morbidity and mortality.

Keywords: Serotonin, Toxicity, Serotonin antagonists.

\footnotetext{
${ }^{1}$ Centro Universitário FIPMoc (UNIFIPMOC), Montes Claros - MG.

*E-mail: laryssadelacerdarod@gmail.com

${ }^{2}$ Faculdade de Ciências Médicas e da Saúde de Juiz de Fora (SUPREMA), Juiz de Fora - MG.

${ }^{3}$ Faculdade da Saúde e Ecologia Humana (FASEH), Vespasiano - MG.

${ }^{4}$ Pontifícia Universidade Católica de Minas Gerais (PUC-MG), Betim - MG.

${ }^{5}$ União das faculdades dos Grandes Lagos (UNILAGO), São José do Rio Preto - SP.

${ }^{6}$ Universidade Federal do Maranhão (UFMA), São Luís - MA.
} 


\section{RESUMEN}

Objetivo: Aclarar la clínica del síndrome serotoninérgico y permitir así su correcto diagnóstico, de manera que su manejo se realice de manera temprana, dando como resultado una prevención significativa de la morbilidad y mortalidad de este síndrome. Revision bibliográfica: El síndrome serotoninérgico se caracteriza por una manifestación adversa del fármaco generada por el aumento de los niveles de serotonina en el sistema nervioso central y periférico. Debido a su falta de manifestaciones específicas, al rápido empeoramiento clínico y al aumento de la prescripción de fármacos serotoninérgicos en las últimas décadas, se hizo necesario formular criterios de diagnóstico para una rápida identificación y reversión. Sin embargo, existe una falta de conocimiento médico sobre dicho síndrome, lo que corrobora su subregistro, lo que hace que este estudio sea relevante. Consideraciones finales: Esta investigación reveló que uno de los desafíos con respecto al síndrome antes mencionado es evitar su diagnóstico incorrecto, por lo tanto, conocer los pilares de su clínica, a través de la educación médica continua, así como las principales interacciones farmacológicas que culminan en la enfermedad, es fundamental para obtener el diagnóstico correcto y, por lo tanto, comenzar las medidas terapéuticas recomendadas temprano, reduciendo la morbilidad y la mortalidad.

Palabras clave: Serotonina, Toxicidad, Antagonistas de la serotonina.

\section{INTRODUÇÃO}

A Síndrome Serotoninérgica (SS) trata-se de uma reação adversa medicamentosa potencialmente fatal que consiste no aumento dos níveis de serotonina no sistema nervoso central e no periférico, gerando uma perda da homeostase corporal, com a tríade clássica de disfunção autonômica, excitação neuromuscular e alteração no estado mental. Suas principais causas estão associadas ao uso de medicamentos com ação nos receptores serotoninérgicos, principalmente quando ocorre o aumento da dosagem terapêutica, associação de dois ou mais desses medicamentos, interações medicamentosas que inibem o catabolismo da droga pelas enzimas P-450 e overdose por substâncias lícitas ou ilícitas que tem efeito serotoninérgico (WANG RZ, et al., 2016; BARTLETT D, 2017).

Tendo em vista que a serotonina atua tanto no sistema nervoso central como no periférico, as alterações clínicas tendem a ser sistêmicas e pouco características. As manifestações mais comuns são: hiperatividade neuromuscular (tremor, clônus muscular, hiperreflexia, rigidez muscular e sinal de Babinski bilateral), hiperatividade do sistema nervoso autonômico (hipertensão, taquicardia, taquipnéia, hipertermia, vômito, diarreia, arritmia e tremor) e alterações do estado mental (agitação, ansiedade, desorientação, inquietação e excitação). O sinal de Babinski configura-se como um dos elementos semiológicos mais relevantes do sistema nervoso e representa a abolição do reflexo cutâneo-plantar, que pode ocorrer quando há bloqueio do arco reflexo e, algumas vezes, no início de uma lesão da via piramidal. Dessa forma, indica lesão da via piramidal ou corticoespinal (PORTO CC, 2014).

Levando isso em consideração, e por ser potencialmente fatal e com rápida evolução, o rápido diagnóstico da síndrome serotoninérgica é essencial para uma boa sobrevida do paciente. Logo, foram desenvolvidos diversos critérios diagnósticos, associando as alterações clínicas com o uso de medicamentos que atuam em receptores de serotonina (BALDO BA e ROSE MA, 2019; BEAKLEY BD, et al., 2015).

Nas últimas décadas, devido ao maior adoecimento da população e do hábito da prescrição indiscriminada de medicamentos existente entre alguns profissionais da saúde, se tornaram também numerosas as prescrições de medicamentos com ação nos receptores de serotonina. Esse fato aliado ao aumento do consumo de substâncias ilícitas psicoestimulantes atuantes nesses receptores pela população, em geral, torna necessário que o conhecimento dessa síndrome seja ainda mais difundido entre os clínicos. Os estímulos mais frequentes da SS são os antidepressivos. Nos Estados Unidos, a incidência do uso desses medicamentos em adultos passou de 6\% em 1999 para 10,4\% em 2010.

Ademais, a ingestão relatada de inibidores seletivos da recaptação de serotonina (ISRSs) cresceu quase 15\% de 2002 a 2005. A síndrome da serotonina pode ser causada por analgésicos, antibióticos, antidepressivos, antieméticos, medicamentos anti-enxaqueca, drogas de abuso, ervas suplementares, 
inibidores de monoamina oxidase e medicamentos de venda livre. Quando causada pelo uso terapêutico de um medicamento serotoninérgico, geralmente ocorre após o aumento das doses, logo após o início do regime de medicação, quando dois ou mais medicamentos serotoninérgicos estão sendo usados juntos ou quando uma interação medicamentosa inibe o metabolismo de um medicamento serotoninérgico por enzimas do citocromo P-450.

Também pode ocorrer após a ingestão de uma única dose de um medicamento serotoninérgico ou exposição única a uma droga ilícita que tenha efeitos serotoninérgicos. Sendo assim, saber quais drogas de abuso, medicações e suplementos podem causar a SS no paciente, tal como compreender os mecanismos pelos quais estes agem no organismo, é de suma importância no momento da prescrição de medicamentos.

Contudo, o grande espectro de apresentações clínicas, quando somado ao conhecimento superficial ou até total desconhecimento por parte dos profissionais de saúde, corrobora para uma subnotificação dessa doença na literatura médica, uma vez que seus graus manifestação variam dos mais leves, que podem passar despercebidos ou serem confundidos com ansiedade, aos mais graves, rapidamente fatais. Sendo assim, torna-se essencial sua elucidação para a classe médica (JUREK L, et al., 2019; BARTLETT D, 2017; FRANCESCANGELI J, et al., 2019; ASUSTA HB, et al., 2019).

É importante destacar que a síndrome da serotonina é autolimitada e pode ser resolvida dentro de 24 a 72 horas após a descontinuação do agente causal, sendo que a falha em reconhecê-la em tempo hábil aumenta a chance de uma evolução para uma forma séria da doença (ASUSTA HB, et al. 2019; JUREK L, et al., 2019). Em vista disso, o presente artigo tem como finalidade esclarecer a clínica da síndrome serotoninérgica e assim possibilitar seu correto diagnóstico, de forma que seu manejo seja feito de maneira precoce, resultando na prevenção significativa da morbimortalidade dessa síndrome.

\section{REVISÃO BIBLIOGRÁFICA}

\section{Epidemiologia}

Segundo dados do Relatório Anual 2013 do Sistema Nacional de Dados de Envenenamento da Associação Americana de Centros de Controle de Intoxicações foram notificadas 55.478 casos de exposição a inibidores da recaptação da serotonina (ISRSs) e inibidores da recaptação da serotonina-noradrenalina, em leitos hospitalares, com 119 exposições fatais, geralmente associadas à ingestão simultânea de outras substâncias. Outro relatório estimou a incidência da SS em pacientes que usaram doses de ISRSs acima do recomendado em aproximadamente $14 \%$, além disso, foi constatado que a taxa de mortalidade nesses pacientes é aproximadamente de $2 \%$ a $12 \%$ (NEW AM, et al., 2015).

A incidência da SS vem crescendo nos últimos anos, reflexo do aumento da oferta de medicamentos moduladores serotoninérgicos disponíveis e do seu uso na prática clínica, em decorrência da elevada prevalência de pacientes com os mais variados transtornos de ansiedade. A incidência relatada também pode refletir uma crescente conscientização diagnóstica da síndrome, visto que muitos casos não são reconhecidos, de modo que a real incidência da síndrome de serotonina ainda é incerta. Outro ponto a ser considerado é que o uso prolongado de antidepressivos aumentou de forma desproporcional em adultos de meia-idade e idosos.

Intuitivamente, como o risco de depressão aumenta dramaticamente em pacientes com comorbidades crônicas, a síndrome da serotonina parece ser mais prevalente em idosos. Além disso, pacientes com múltiplas comorbidades tomam mais medicamentos, aumentando o risco de polifarmácia e reações adversas a medicamentos. Embora a epidemiologia da síndrome ainda não tenha sido extensivamente estudada, a combinação de idade e comorbidades pode aumentar o risco para o desenvolvimento dessa condição (ABLES AZ e NAGUBILLI R, 2010).

A síndrome da serotonina é bem descrita na literatura médica, mas as informações disponíveis são de relatos de casos e de pequenas séries de casos, dessa forma, a incidência da síndrome não é totalmente conhecida e as recomendações de tratamento foram derivadas empiricamente de dados relativamente antigos. Essas incertezas, juntamente com a dificuldade em identificar uma condição que ainda não possui 
um teste confirmatório específico, resultando em subnotificação, apresentam desafios importantes para determinar sua incidência de forma precisa. Apesar da incerteza de números precisos, é evidente que o número de casos está aumentando, o que reflete o crescente número de drogas serotoninérgicas disponíveis e o aumento do uso desses agentes na prática clínica (BARTLETT D, et al., 2017; ABLES AZ e NAGUBILLI $R, 2010)$.

\section{Fisiopatologia}

As células dos núcleos da rafe localizadas na linha mediana do tronco cerebral (do mesencéfalo à medula) são a fonte da 5-hidroxitriptamina ou serotonina (5-HT) neuronal no Sistema Nervoso Central (SNC). Os 9 núcleos rafe (B1-B9) estão centrados na formação reticular, e os axônios dos neurônios serotoninérgicos dessa região formam um sistema de neurotransmissores que afeta funcionalmente a maior parte do SNC.

O grupo rostral de núcleos se projeta em múltiplas estruturas corticais e subcorticais, contribuindo para a regulação da vigília, atenção, comportamento afetivo (depressão e ansiedade), comportamento sexual, apetite, termorregulação e enxaqueca.

O grupo caudal se projeta para as regiões da coluna vertebral e está envolvido no tônus motor e na nocicepção. Na periferia, o 5-HT, produzido nas células enterocromafins (EC) do trato gastrointestinal, está envolvido na regulação da motilidade gastrointestinal, bem como em vários papéis na biologia vascular. Isso inclui o controle da pressão arterial por vasoconstrição ou vasodilatação, dependendo do tipo de receptor expresso na parede do vaso, bem como o controle da hemostasia e da função plaquetária (SCOTTON WJ, et al.,2019).

A serotonina afeta uma família de receptores que possui sete membros, subtipos dos 5 -hidroxitriptamina ou serotonina (5-HT), como os 5-HT1A e 5-HT2A, que são frequentemente os mais acometidos em quadros da síndrome serotoninérgica. Esses receptores se relacionam à regulagem de diversos processos fisiológicos como sono, reprodução, comportamento alimentar, emoção, cognição e comportamento social. Dessa forma o uso das medicações que atuam neles ocorre com frequência, assim como os quadros relacionados a intoxicação. As condições que podem alterar a regulação da serotonina incluem uso de medicações em doses terapêuticas, interações medicamentosas, overdoses intencionais ou não intencionais e transições sobrepostas entre medicamentos.

Um notável número de medicamentos de diferentes classes tem sido apontado como causadores da síndrome da serotonina. Embora os mais comumente culpados sejam os inibidores da monoamino-oxidase (IMAOs), inibidores da recaptação de serotonina-noradrenalina (IRSNs) e inibidores seletivos da recaptação de serotonina (ISRSs), a lista de potenciais contribuintes é longa e inclui substâncias frequentemente negligenciadas e tidas como inócuas, como ervas e medicamentos lícitos (BEAUDOIN-GOBERT M e SGAMBATO-FAURE V, 2014; WANG RZ, et al., 2016; FOONG AL, et al., 2018).

Atualmente, os ISRSs são os medicamentos antidepressivos mais comumente prescritos e, consequentemente, são os mais frequentemente relacionados à toxicidade serotoninérgica. Estima-se que $15 \%$ das overdoses com ISRSs levam à toxicidade leve ou moderada da serotonina. Os agentes serotoninérgicos utilizados em conjunto podem aumentar o risco de síndrome serotoninérgica grave; um ISRS e um IMAO, em conjunto, representam o maior risco. Os SSRIs todos têm um mecanismo de ação semelhante, seletivamente bloqueando as bombas de absorção de serotonina, que levam a um aumento na disponibilidade desse neurotransmissor.

A seletividade dos SSRIs permite que eles atinjam o efeito desejado (inibição de a bomba de absorção de serotonina) enquanto não tem efeito em outras proteínas transportadoras (como aqueles associados à norepinefrina ou dopamina).

Paralelamente Inibidores de monoamina oxidase, inicialmente utilizados como antidepressivos e hoje difundidos pelo seu valor terapêutico em várias condições neurodegenerativas comuns como Parkinson's e Alzheimer, atuam inibindo a ação da enzima monoaminaoxidase que catalisa a degradação da norepinefrina, dopamina e serotonina cerebral (WANG RZ, et al., 2016, LOCHMAN D e RICHARDSON T, 2018; SEAN CG, et al., 2016). 
A serotonina ou 5-hidroxitriptamina (5HT) é um neurotransmissor sintetizado no corpo e é regulado por múltiplos mecanismos homeostáticos. O excesso de serotonina é eliminado pelo endotélio pulmonar ou hepático. Portanto, em situações de dano endotelial periférico ou pulmonar, a capacidade de metabolizar o excesso de serotonina pode ser prejudicada. Essa alteração do metabolismo da serotonina, em combinação com o uso de medicamentos serotoninérgicos, pode contribuir para o desenvolvimento da síndrome da serotonina em pacientes críticos. Na Unidade de Terapia Intensiva (UTI), a síndrome da serotonina está tipicamente associada ao uso de duas ou mais medicações serotoninérgicas concomitantes, embora também tenha sido relatada em monoterapia (NEW AM, et al., 2015) (Figura 1).

Figura 1 - Mecanismo fisiopatológico da síndrome serotoninérgica.

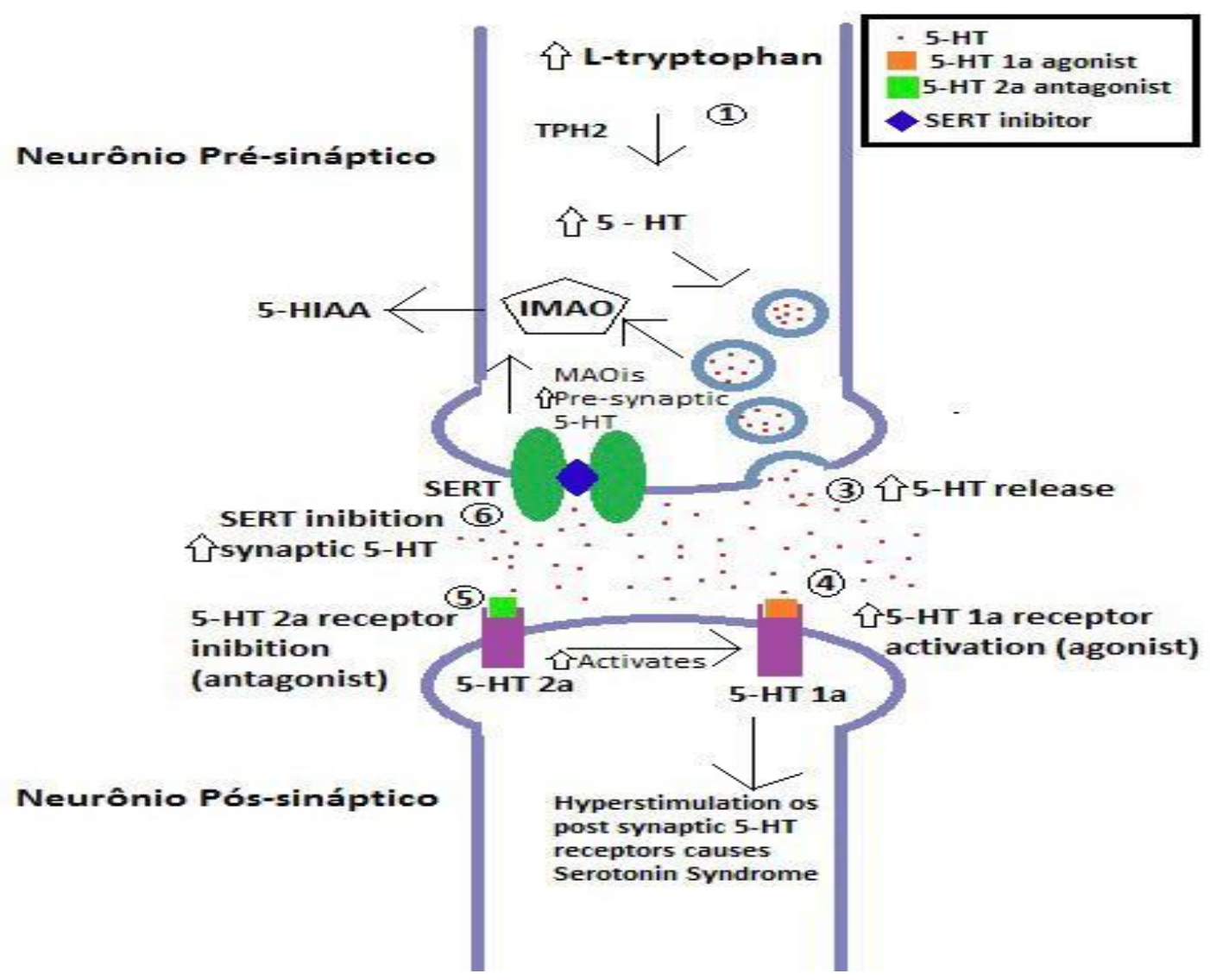

Legenda: (1) Ocorre o aumento dos níveis de L-triptofano, provocando aumento endógeno do neurotransmissor 5-HT, etapa catalisada pela enzima Triptofano hidroxilase 2 (TPH2). (2) Aumento da concentração pre-sináptica de 5-HT devido a inibição do metabolismo serotoninérgico pelos MAOIs. (3) liberação excessiva de 5-HT devido a medicamentos, principalmente as anfetaminas e seus derivados: MDMA, cocaína e levodopa. (4) Agonismo direto ou indireto dos receptores pós-sinápticos 5-HT1A. (5) Antagonismo direto ou indireto dos receptores $5 \mathrm{HT} 2 \mathrm{~A}$, auxiliando na hiperestimulação pós sináptica dos receptores de $5-\mathrm{HT}$. (6) Aumento sináptico dos níveis de 5-HT devido à inibição do SERT, gerado pelos inibidores da recaptação, como ISRSs. MAOIs indica inibidores da monoamino-oxidase (IMAOs); MDMA, 3,4-metilenodioxi-metanfetamina; SERT, proteína transportadora da recitação de serotonina; ISRSs, Inibidores seletivos de receptação de serotonina.

Fonte: RODRIGUES LL, et al., 2020.

\section{Manifestações clínicas}

As características clínicas clássicas da intoxicação à serotonina incluem uma combinação de alteração do estado mental, instabilidade autonômica e hiperatividade neuromuscular. A intensidade dos achados clínicos reflete o grau de toxicidade da serotonina (FOONG AL, et al., 2018; ADRIENNE ZA e NAGUBILLI R, 2010). 
Por não possuir pródromos específicos e as manifestações serem também inespecíficas, a síndrome da serotonina pode ser facilmente negligenciada ou exacerbada se não for cuidadosamente avaliada. $O$ diagnóstico requer um limiar baixo de suspeição e uma história meticulosa, além do exame físico completo. Seu diagnóstico tardio deve ser evitado, pois a SS causa alta morbimortalidade se não for identificada e tratada rapidamente. Por isso, o reconhecimento precoce é necessário para interromper a ação dos agentes serotoninérgicos responsáveis, e iniciar medidas terapêuticas, adaptadas a cada paciente (FOONG AL, et al., 2018; FRANK C, 2008; JUREK L, et al., 2018).

\section{Diagnóstico}

No cenário da emergência, o rápido e correto diagnóstico da Síndrome Serotoninérgica (SS) é fator determinante uma vez que esta pode cursar com uma alta morbimortalidade se os agentes serotoninérgicos não forem rapidamente interrompidos Primeiramente, deve-se ter conhecimento dos medicamentos de uso diário do paciente, considerando-se que aqueles mais relacionados à ocorrência da síndrome são os Inibidores da Monoamina Oxidase (IMAO), os IRSS e o Tramadol (agonista do receptor $\mu$-opióide e Inibidor da recaptação da Serotonina e Noradrenalina).

Além disso, caso o paciente tenha feito ingestão de dose exacerbada associada ao uso de inibidores serotoninérgicos do citocromo P450 se torna maior a chance de apresentar SS e convulsões. Nesse sentido, Familiares e Enfermeiros de cuidados intensivos desempenham um papel fundamental na identificação desse quadro, pois podem ajudar a reconhecer ao coletar a história clínica do paciente, atentando-se tanto aos medicamento em uso quanto aos sintomas específicos e gerais (JUREK L, et al., 2018; FOONG AL, et al., 2018; NEW AM, et al., 2015).

Uma vez que os sintomas da crise podem variar muito, foram desenvolvidos os critérios de Radomski, para facilitar a identificação da síndrome, o qual classificou os sintomas em critério maiores, sendo estes confusão mental, coma, hipertermia, sudorese, mioclonia, hipertonia, hiperreflexia e tremor, e em critérios menores, hiperatividade, agitação, insônia, taquicardia, taquipneia, dispneia, hipotensão ou hipertensão arterial, diarreia, incoordenação, midríase, acatisia e ataxia. Segundo esse critério há confirmação da síndrome serotoninérgica se houverem 4 critérios maiores ou 3 maiores e 2 menores.

Sendo assim, a apresentação característica inclui uma tríade de disfunção autonômica, alteração de estado mental e excitação muscular. Frisa-se, contudo, que nem todos os pacientes apresentarão estes sintomas simultaneamente, por isso, também é fundamental considerar que a síndrome se manifesta com um início abrupto em até 24 horas após o uso de dose elevada ou introdução de um novo medicamento (BIRMES P, et al., 2003; WANG RZ, et al., 2016; CHRISTENSEN RC, et al., 2005).

Paralelamente, o profissional inserido no sistema primário deve atuar identificando o uso excessivo de medicações de ação serotoninérgica, possuindo um cuidado especial com pacientes que têm certas medicações em seu plano terapêutico, como citalopram em associação com moclobemida, buspirona, trazodona e lítio, visto que essas drogas podem aumentar significativamente a probabilidade de ocorrência da síndrome (FRANK C, 2008; TALARICO G, et al.,2011).

\section{Diagnóstico diferencial}

Um importante diagnóstico diferencial se faz com a Síndrome Neuroléptica Maligna (SNM), pois ambas são condições relativamente raras, com risco de vida a reações adversas de medicamentos psicotrópicos. Sintomas como alteração mental, distúrbios autonômicos, incluindo hipertermia, taquicardia, taquipneia, variações na Pressão arterial sistêmica e hiper excitabilidade neuromuscular são características proeminentes de ambas as síndromes.

As características clínicas distintivas mais importantes incluem hiperreflexia, mais acentuada nos membros inferiores, clone ocular e tremores na SS, enquanto a SMN é uma síndrome bradicinética caracterizada, principalmente, por hiporreflexia. Além disso, os primeiros sintomas da SS são frequentemente observados nas primeiras 24 horas após o uso dos agentes serotoninérgicos e o quadro clínico é resolvido, normalmente, alguns dias após o início do tratamento e a omissão do medicamento. Por outro lado, os sintomas característicos da SNM podem ocorrer mais tardiamente e é esperado que o quadro regride entre 9 e 14 dias. 
Quanto aos exames laboratoriais, embora não haja teste de diagnóstico para nenhuma das síndromes, níveis elevados de creatina quinase, distúrbios eletrolíticos, especialmente hipocalcemia, baixos níveis de ferro, leucocitose e proteinúria são mais característicos de pacientes com SNM. A principal medida para o manejo de ambas as síndromes é a cessação do fármaco desencadeador. Ao contrário da síndrome neuroléptica maligna, a síndrome da serotonina não deve ser considerada uma reação idiossincrática extremamente rara à medicação, mas sim uma progressão da toxicidade serotoninérgica com base no aumento dos níveis que podem ocorrer em qualquer paciente, independentemente da idade (DOSI R, et al., 2014).

\section{Terapêutica}

O manejo da Síndrome Serotoninérgica se assenta em dois pilares: a retirada dos medicamentos serotoninérgicos ofensivos e a prestação de cuidados de suporte, como: fluidos intravenosos, oxigênio suplementar, telemetria e avaliações neurológicas. Ficou evidente após a interpretação dos estudos que a maior parte dos casos leves da SS desaparecerá após o início da terapia e a interrupção dos medicamentos serotoninérgicos. Dos pacientes portadores da SS $70 \%$ se recuperam completamente com $24 \mathrm{~h}, 40 \%$ necessitam de admissão em uma unidade de terapia intensiva e apenas $25 \%$ necessitam intubação orotraqueal (WANG RZ, et al., 2016; ADRIENNE ZA e NAGUBILLI R, 2010; TORMOEHLEN LM e RUSYNIAK DE, 2018; BARTLETT D, 2017).

Nos presentes estudos de BALDO BA e ROSE (2020), juntamente com DVIR Y and SMALLWOOD P (2007) foi evidenciado que a abordagem terapêutica depende da gravidade da doença. Sendo assim, casos leves podem ser tratados com a descontinuidade da oferta da droga, suporte terapêutico com fluidos intravenosos para hidratação e facilitação da diurese e tratamento com benzodiazepínicos para prevenir agitação. Por sua vez, casos moderados necessitam de terapêutica mais agressiva da instabilidade autonômica e deve-se considerar o tratamento com um antagonista da serotonina (BOYER EW et al.,2020).

Em pacientes com severa toxicidade serotoninérgica está indicado a sedação e a intubação orotraqueal, sendo nesses pacientes a hipertermia e a rigidez as duas principais preocupações. Contudo é importante ressaltar que as evidências da literatura demonstram que os antipiréticos não são úteis na redução da temperatura nesses quadros, mas sim os benzodiazepínicos que diminuem a temperatura por reduzirem a contração muscular e a hiperatividade autonômica (BARTLETT D, et al., 2017; WANG RZ, et al., 2016; TORMOEHLENL M e RUSYNIAK DE, 2018).

A farmacoterapia dirigida envolve a administração de antagonistas dos receptores 5-HT. A CiproHeptadina é um anti-histamínico com propriedades antagonísticas inespecíficas de 5-HT1A e 5-HT2A. Recomenda-se que uma dose inicial de $12 \mathrm{mg}$ seja considerada, seguida de $2 \mathrm{mg}$ adicionais a cada duas horas se os sintomas persistirem. Após a estabilização do paciente, uma dose de manutenção de $8 \mathrm{mg}$ a cada seis horas pode ser administrada. Apesar dessa medicação ser amplamente utilizada, em unanimidade os autores deixam clara a necessidade de novos estudos para comprovar sua efetividade, assim como ocorre com outras terapias com uso das drogas Clorpromazina e Olanzapina, utilizadas eventualmente por seus efeitos 5-HT antagonistas. Por outro lado, terapias descritas, como o uso de propranolol, bromocriptine e dantrolene não são recomendadas (ADRIENNE ZA e NAGUBILLI R, 2010; WANG RZ, et al., 2016; NEW AM, et al., 2015; BOYER EW e SHANNON M, 2005).

Pacientes com sintomas como hipertensão e taquicardia, comuns na SS, podem ser tratados com esmolol ou antihipertensivos como o nitroprussiato. Por outro lado, medidas como restrições físicas são desaconselhadas e podem contribuir para a mortalidade ao aplicar contrações musculares isométricas associadas à acidose láctica grave e hipertermia. Os autores enfatizam que uma vez que o suporte terapêutico foi iniciado e os pacientes foram tratados adequadamente o prognóstico é bom, com raras fatalidades e expectativa de total recuperação (BOYER EW et al., 2020; BARTLETT D, 2017; WANG R, et al., 2016).

O manejo das consequências dessa interação medicamentosa inclui administrar um benzodiazepínico, como o lorazepam, tratamentos de suporte para evitar lesões e descontinuar o tramadol e outros agentes serotoninérgicos pró- convulsivantes. Pode-se ainda considerar uma eletroencefalografia e ressonância magnética cerebral para detectar foco de ataque subjacente ou anormalidade estrutural, e na ausência de 
patologia subjacente específica, medicamento antiepilético profilático não são indicados (HASSAMALI S, et al., 2018).

\section{CONSIDERAÇÕES FINAIS}

Diante os atuais desafios proporcionados pelo aumento do uso de medicações que atuam nos receptores da serotonina, principalmente com aumento dos quadros de depressão e ansiedade, chega-se ao entendimento de que a forma mais plausível de prevenção da SS é a disseminação sobre seu conhecimento no meio médico e acadêmico, como forma de prevenir, identificar e tratar de maneira adequada. Frente a essa perspectiva esse estudo propõe a necessidade de capacitação dos profissionais de saúde para que haja abordagem e manejo correto da síndrome, por se tratar de um quadro potencialmente fatal quando não conduzido de forma correta. Dessa maneira, os estudos abordados por esse trabalho evidenciaram que 0 conhecimento das causas e efeitos da SS são indispensáveis, tanto para os profissionais da emergência, quanto para os profissionais da atenção primária, que devem estabelecer um plano terapêutico consciente, junto ao paciente como forma prevenção e alerta dessa iatrogenia.

\section{REFERÊNCIAS}

1. ABLES AZ, NAGUBILLI R. Prevention, recognition, and management of serotonin syndrome. Am Fam Physician, 2010; 81(9):1139-1142.

2. ASUSTA HB, et al. Serotonin Syndrome in Obstetrics: A Case Report and Review of Management. Military Medicine, 2019 Jan 1; 184(1-2):284-286.

3. BALDO BA, ROSE MA. The anaesthetist, opioid analgesic drugs, and serotonin toxicity: a mechanistic and clinical review. British Journal of Anaesthesia, 2020;124(1):44- 62.

4. BARLETT D. Drug-Induced Serotonin Syndrome. Critical Care Nurse, 2017;37(1): 49-54.

5. BEAUDOIN-GOBERT M e SGAMBATO V. Serotonergic pharmacology in animal models: From behavioral disorders to dyskinesia. Neuropharmacology, 2014; 81, 15-30.

6. BEAKLEY BD, et al. Tramadol, Pharmacology, Side Effects, and Serotonin Syndrome: A Review. Pain Physician, 2015;18(4): 395-400.

7. BIRMES P, et al. Serotonin syndrome: a brief review. CMAJ, 2003; 168 (11): 1439-42.

8. BOYER EW, SHANNON M. A Síndrome da Serotonina. N Engl J Med 2005; 352(11): 1112-1120

9. CHRISTENSEN RC. Identifying serotonin syndrome in the emergency department. The American Journal of Emergency Medicine, 2005; 23(3), 406-408.

10. DOSI R, et al. Serotonin syndrome versus neuroleptic malignant syndrome: a challenging clinical quandary. BMJ Case Rep 2014; 2014: $1-4$.

11. DVIR Y, SMALLWOOD P. Serotonin syndrome: a complex but easily avoidable condition. General Hospital Psychiatry, 2008; 30(3), 284-287.

12. FOONG AL, et al. Demystifying serotonin syndrome (or serotonin toxicity). Can Fam Physician, 2018; 64(10):720727.

13. FRANCESCANGELI J, et al. The Serotonin Syndrome: From Molecular Mechanisms to Clinical Practice. International Journal of Molecular Sciences, 2019; 20(9): 1-17.

14. FRANK C. Recognition and treatment of serotonin syndrome.Can Fam Physician, 2008; 54(7):988-992.

15. JUREK $L$, et al. Le syndrome sérotoninergique: une revue actualisée de la littérature. La Revue de Médecine Interne, 2019; 40(2):98-104.

16. NEW AM, et al. Psychiatric Emergencies in the Intensive Care Unit, AACN Advanced Critical Care, 2015; 26(4): 285293.

17. PORTO CC. Semiologia médica. $7^{\text {ạ }}$ ed. Rio de Janeiro: Guanabara Koogan, 2014; 1204p.

18. SAMEER HASSAMAL, et al.Tramadol: Understanding the Risk of Serotonin Syndrome and Seizures, The American Journal of Medicine, 2018;131(11): 1-20.

19. SCOTTON WJ, et al. Serotonin syndrome: pathophysiology, clinical characteristics, treatment and possible future guidelines. International Journal of Tryptophan Research, 2019; 12:1-14

20. SEAN CG, et al. The role of monoamine oxidase A in aggression: current translational developments and future challenges. Prog Neuropsychopharmacol Biol Psychiatry, 2016; 69: 90-100

21. TALARICO G, et al. Serotonin toxicity: a short review of the literature and two case reports involving Citalopram. Neurol Sci, 2011; 32(3): 507-509.

22. TORMOEHLEN LM, RUSYNIAK DE. Neuroleptic malignant syndrome and serotonin syndrome. Handbook of Clinical Neurology, 2018; 157:663-675.

23. WANG RZ, et al.Serotonin syndrome: Preventing, recognizing, and treating it. Cleveland Clinic Journal of Medicine, 2016; 83(11), 810-817. 